\title{
THE PULSATIONS AND EVOLUTION OF AGB STARS IN THE LARGE MAGELLANIC CLOUD
}

\author{
Z. G. GONG AND Y. LI \\ Yunnan Observatory, Kunming, P.R. China
}

\begin{abstract}
The evolutionary status and pulsational characteristics of AGB stars in the LMC have been studied. It is found that only those stars with a small mixing-length ratio can fit the observed period-luminosity relation. Strong non-adiabatic effects were found in the outer envelope and must be taken into account in future work.
\end{abstract}

\section{Introduction}

The long-period variable stars (LPVs) are red variables which include the Mira stars, semiregular variable stars, red supergiant variable stars, and periodically pulsating $\mathrm{OH} / \mathrm{IR}$ sources whose periods are from several tens of days to more than one thousand days (Hughes 1989; Hughes \& Wood 1990; Wood, Moore \& Hughes 1991; Wood et al. 1992; Whitelock 1995). As all of these stars located in the red and bright part of the HR diagram, their study will be very important for the understanding of late stages of stellar evolution.

The LPVs in theoretical astrophysics are classified into two subgroups. One is for those red supergiant LPVs and some OH/IR sources which are thought to be massive stars in their late helium-burning stages ( $\mathrm{Li} \&$ Gong 1994). The other group includes the intermediate-mass stars on the asymptotic giant branch (AGB), including Miras, semiregular variables, and some other $\mathrm{OH} / \mathrm{IR}$ sources. In this paper we will focus on the latter subgroup (AGB stars).

As the stars in the Large Magellanic Cloud (LMC) are all at almost the same distance from our observers, and the AGB LPVs in the LMC have been so well studied that a lot of data are available, we will pay most attention to these AGB LPVs. 
Theoretical studies of the characteristics of AGB LPVs can be found in some early papers. From these publications we find that, although many aspects of the evolutionary and pulsational status of these stars are known in general, problems and discrepancies still remain, especially as regards the pulsation mode. In the work of Fox \& Wood (1982) we can find a full set of pulsational properties of AGB LPVs, but their introduction of a convection - pulsation interaction mechanism, which has not been completely solved and has not been widely used in investigations of other variable stars, in their stellar pulsation calculation is still questionable. And their suggestion of the AGB LPVs being first-overtone pulsators is different from the later conclusion of Ostlie \& Cox (1986), who regarded these stars as pulsating in the fundamental mode. The question regarding the work of Ostlie \& Cox is that they artificially increased the opacity of the stars by averaging the opacity of upward and downward cells, and such a treatment would seem to need some physical analysis, which was ignored, before its application.

When nonlinear effects are considered in the pulsation calculation of AGB LPVs, the discrepancy in pulsation mode still exists. In Wood (1990) and Bessell et al. (1996), Miras are thought to pulsate in the fundamental mode, while in Barthès \& Tuchman (1994) they are first-overtone pulsators.

Bowen (1988), on the other hand, did very helpful work on the nonlinear atmospheric activity of Miras and supported the conclusion that AGB LPVs are fundamental-mode pulsators. However, the need of a pulsational inner boundary condition in his computation implied that the connection between envelope pulsation and this boundary condition must be considered. Some information about the interior is very important in the study of the behavior of the atmosphere, too.

In this paper we will investigate both the evolution and pulsation of intermediate-mass AGB LPVs based on recent physics considerations to get some more ideas about these stars. In $\S 2$ we discuss our codes and input physics. In $\S 3$ we compare our results with observation, and then make some comments.

\section{Codes and Input Physics}

We evolved our models from the ZAMS to the AGB by using a modified version of the Kippenhahn et al. (1967) stellar structure and evolution code to get static models of AGB LPVs, then calculated their linear non-adiabatic radial pulsation properties under the $\mathrm{Li}(1992 \mathrm{a}, \mathrm{b})$ stellar pulsation code. In this paper we study intermediate-mass AGB LPVs whose initial masses are $3 M_{\odot}$ and $7 M_{\odot}$. The mass loss rate was calculated following Nieuwenhuijzen \& de Jager (1990), which is valid throughout the HR diagram. The evolutionary tracks of these stars are shown in Figure 1. 


\section{Evolutionary Tracks}

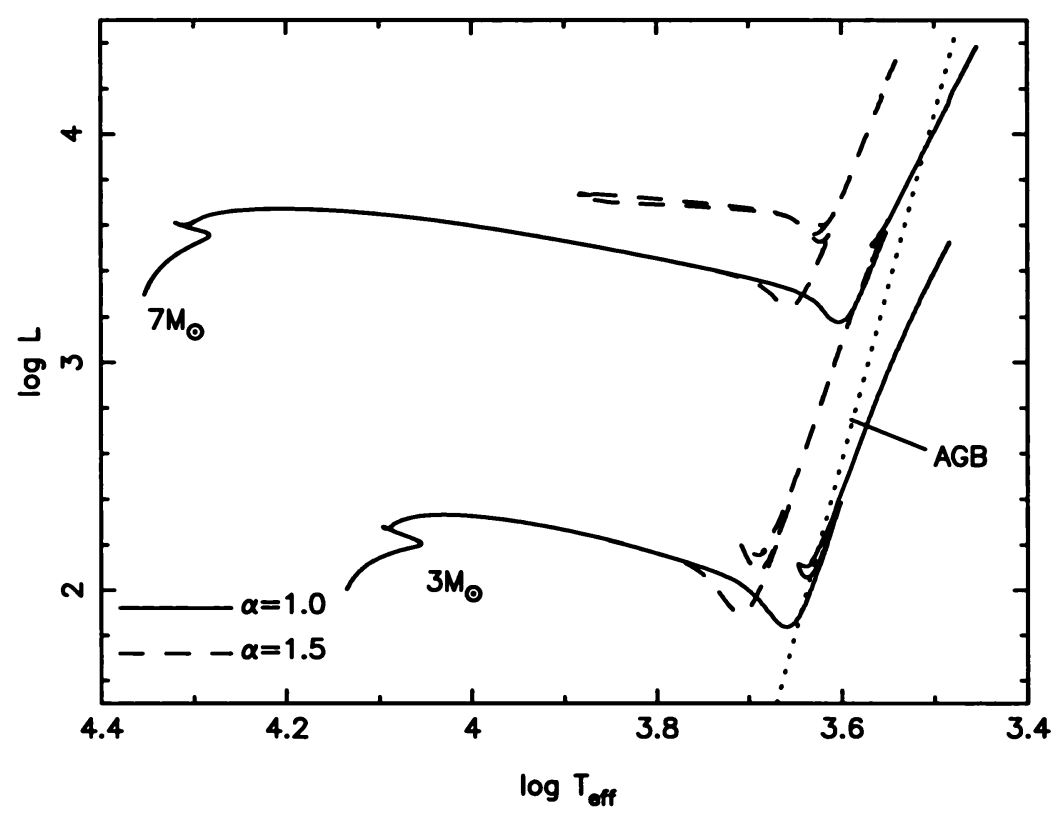

Figure 1. Evolutionary tracks of $3 M_{\odot}$ and $7 M_{\odot}$ stars.

The initial chemical composition was chosen to be $(\mathrm{X}, \mathrm{Z})=(0.7,0.01)$ to fit the requirements of stars in the LMC, whose heavy element abundance is thought to be only one-half that of the solar neighborhood. Recent available opacities are used in our evolution calculation, in which OPAL opacities (Rogers \& Iglesias 1992) are used in the high-temperature region $(\log T>$ 3.85), while those of Alexander \& Ferguson (1994), which include both atomic and molecular opacities, are used in the low-temperature region $(\log T \leq 3.85)$. We find that both the data and their derivatives can connect smoothly at this border.

Convective energy transportation was treated according to the mixing length theory of Böhm-Vitense (1958) with the Schwarzschild criterion. The temperature gradient, unlike the treatment of Kippenhahn et al., was calculated by Böhm-Vitense's formalism throughout the whole model, and the effect of overshoot or semiconvection was not included.

In this paper two different pressure scale heights $\alpha=l / H_{p}$ were tested. One was $\alpha=1.5$, which is usually used in stellar evolution calculations and is close to the value in the standard solar model (Guzik \& Cox 1995). The other one was $\alpha=1.0$, which is smaller than in most published evolution studies, but it was found that this small value allows the pulsation mode 


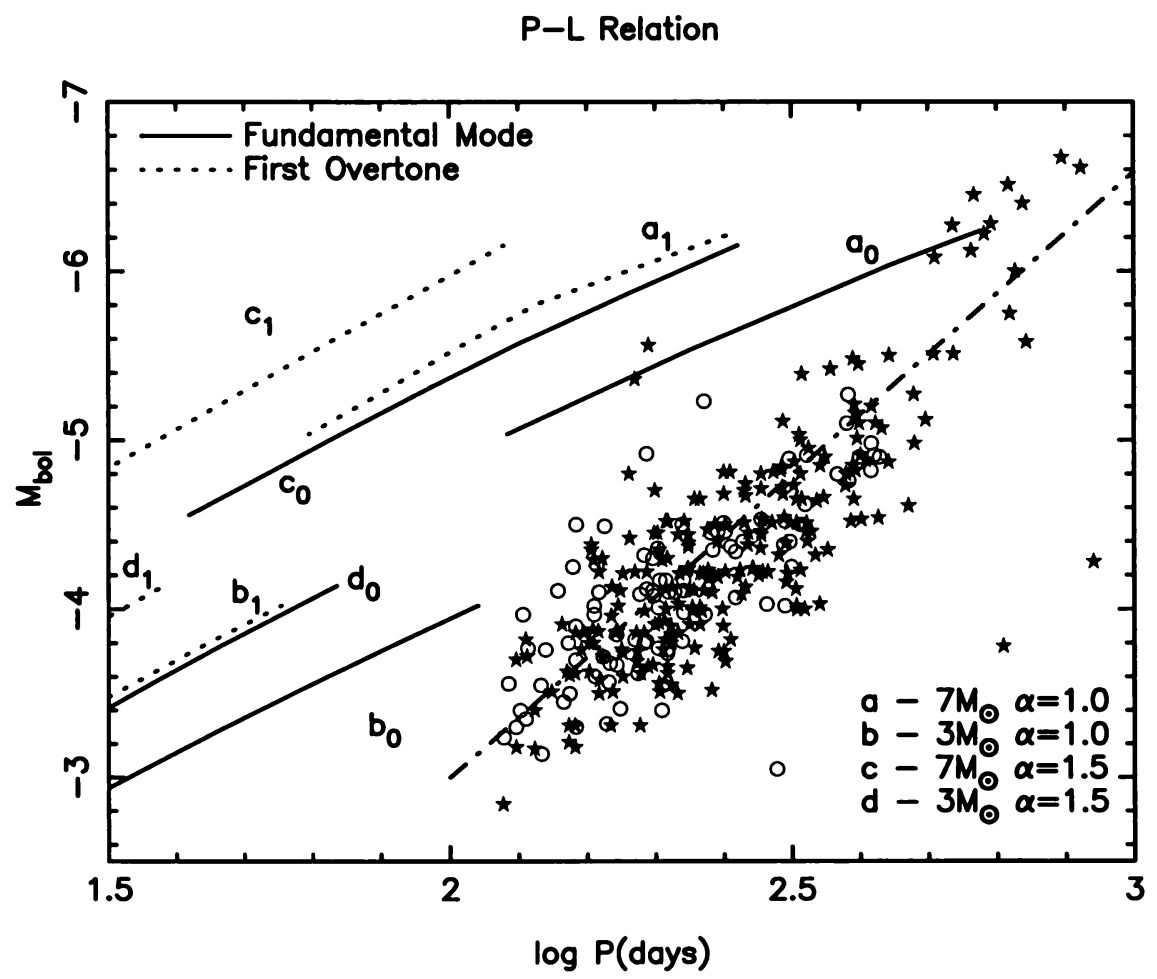

Figure 2. Period-luminosity relation of AGB LPVs in the LMC.

of AGB LPVs to fit the observed data much better.

\section{Results and Discussion}

From our evolution and pulsation calculation it seems that all stars will be pulsationally unstable when they evolve onto the asymptotic giant branch, and the period of such stars will be from several tens of days at the lower AGB to several hundred days at the beginning of thermal pulse phase. The brighter and cooler the stars are, the longer their period will be. This is not difficult to understand because the brighter and cooler stars have a bigger cavity to allow the pulsation wave to travel, and the local sound speed doesn't change very much. Hence, the period will be longer.

When we compare our results with the observational data of AGB LPVs in the LMC (Hughes \& Wood 1990) in Figure 2, we find that in the periodluminosity diagram the first-overtone lines, no matter what the mass and $\alpha$ value, all lie far from the observed data, and they show no potential for reaching the region where the observed stars are located. So we suggest that 


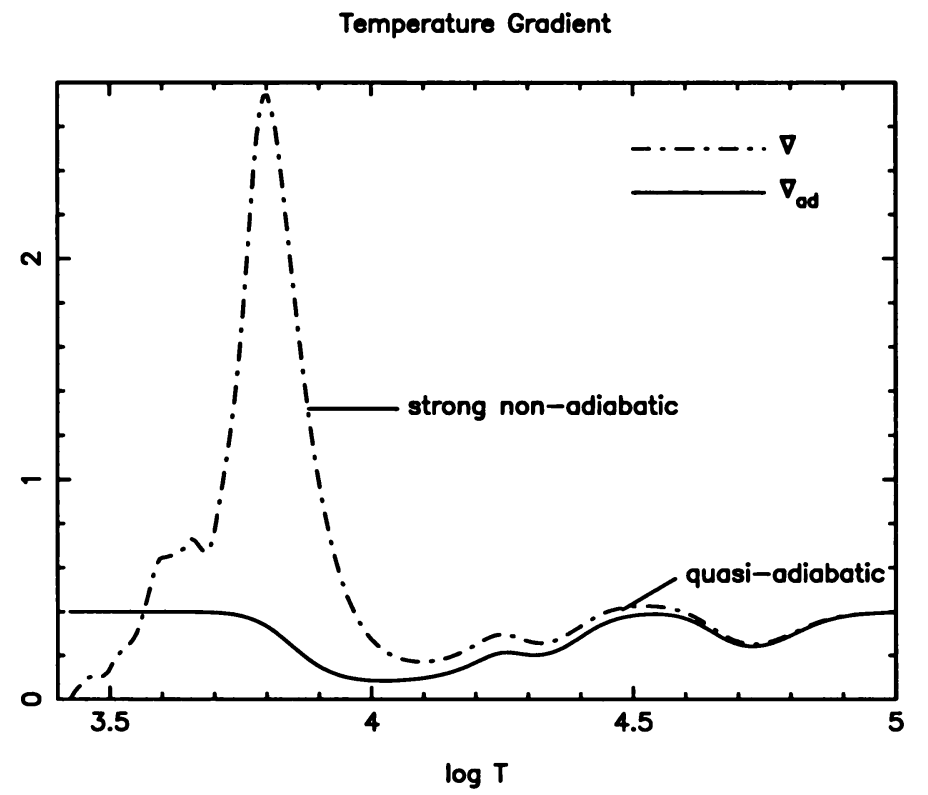

Figure 3. Temperature gradient in the outer envelope of AGB LPVs.

the first overtone is not likely to be the first candidate for the pulsation mode of AGB LPVs in the LMC.

For the fundamental mode things are different. Stars with the bigger $\alpha$ value (c \& d) lie further from the observed values than those with the smaller $\alpha$ value (a \& b). It seems that the fundamental mode of the low $\alpha$ value AGB stars can fit better with the observations. The $b_{0}$ line does not cross the observed star group because our calculation stopped at the beginning of the thermal pulse phase, and when thermal pulses start the star will evolve to be brighter and cooler and its period will be longer so that this line may cross the observed stars. Our conclusion is that the AGB LPVs may possibly be fundamental mode pulsators.

In our stellar pulsation calculation we find that, in the outer envelopes of intermediate-mass AGB stars $(\log T<4.0)$, the pulsation is strongly non-adiabatic (Figure 3). The quasi-adiabatic stellar pulsation theory that is widely used in solving the non-adiabatic effect in stellar pulsation is not valid in this region. A theory which can include the strong non-adiabatic effect is needed if we want to know more accurately the pulsational characteristics in the outer envelopes of cool stars (Li \& Gong 1998).

In conclusion we suggest that AGB LPVs in the LMC are more likely to pulsate in their fundamental mode, and that a small mixing length ratio 
$\alpha$ is required in fitting the theoretical results with the observed stars.

The authors thank Dr. Robert Wing for his fruitful help in preparing this paper, and Dr. Nami Mowlavi for helpful discussion. One of the authors (Z.G.) thanks Dr. Robert Wing for the invitation and grants to attend IAU Symposium 177. This work was supported by the Chinese National Natural Science Foundation.

\section{References}

Alexander, D. R. \& Ferguson, J. W. 1994, ApJ, 437, 879

Barthès, D. \& Tuchman, Y. 1994, $A \& A, 289,429$

Bessell, M.S., Scholz, M. \& Wood, P. R. 1996, $A \& A, 307,481$

Böhm-Vitense, E. 1958, Z. Astrophys., 46, 108

Bowen, G. H. 1988, ApJ, 329, 299

Fox, M.W. \& Wood, P. R. 1982, ApJ, 259, 198

Guzik, J. A. \& Cox, A. N. 1995, ApJ, 448, 905

Hughes, S. M. G. 1989, $A J, 97,1634$

Hughes, S. M. G. \& Wood, P. R. 1990, AJ, 99, 784

Kippenhahn, R., Weigert, A. \& Hofmeister, E. 1967, Meth. Comp. Phys., 7, 129

Li, Y. 1992a, $A \& A, 257,133$

Li, Y. $1992 b, A \& A, 257,145$

Li, Y. \& Gong, Z. G. 1994, $A \& A, 289,449$

Li, Y. \& Gong, Z. G. 1998, in IAU Symp. 181: Sounding the Solar and Stellar Interior, Poster Volume, ed. F. X. Schmider and J. Provost (Nice: Université de Nice), p. 233

Nieuwenhuijzen, H. \& de Jager, C. 1990, $A \& A, 231,134$

Ostlie, D. A. \& Cox, A. N. 1986, $A p J, 311,864$

Rogers, F. J. \& Iglesias, C. A. 1992, ApJ Supp., 79, 507

Whitelock, P. 1995, Astrophys. \& Space Sci., 230, 177

Wood, P.R. 1990, in From Miras to Planetary Nebulae, ed. M.O. Mennessier and A. Omont (Editions Frontières), p. 67

Wood, P. R., Moore, G. K. G. \& Hughes, S. M. G. 1991, in IAU Symp. 148: The Magellanic Clouds, ed. R. Haynes and D. Milne (Kluwer), p. 259

Wood, P. R., Whiteoak, J. B., Hughes, S. M. G., Bessell, M.S., Gardner, F. F. \& Hyland, A. R. $1992, A p J, 397,552$ 